\title{
Magnetisation transfer ratio of normal brain white matter: a normative database spanning four decades of life
}

\author{
N C Silver, G J Barker, D G MacManus, P S Tofts, D H Miller
}

\begin{abstract}
Objectives-To establish a normative database for magnetisation transfer ratio (MTR) measurements in the white matter of healthy adult brains. Such MTR values were evaluated for regional variation and evidence of differences associated with aging, sex, and handedness.

Methods-Forty one healthy volunteers, ranging in age from 16 to 55 years, underwent axial brain magnetisation transfer (MT) imaging on a 1.5 Tesla magnetic resonance scanner. Calculated MT images allowed evaluation of MTR from specific regions within the corpus callosum, cerebral hemispheres, and pons.
\end{abstract}

Results-Highest values were noted in the corpus callosum. No significant sex differences were seen for any region studied. Small but significant age related reductions in MTR were noted in the corpus callosum and other cerebral white matter regions studied. Comparing MTR values between young (16-35 years) and older (36-55 years) age groups, this was most apparent in the corpus callosum $(40.82 \%$ units in the young group $v 40.28 \%$ units in the older group, $P<0.05$ ) and frontal white matter $(39.65 \%$ units in the young group $v 39.18 \%$ units in the older group, $P$ $<0.005)$. In addition, values for MTR were analysed for evidence of hemispheric asymmetry. MTR values were higher in the left hemisphere for all regions studied, reaching significance in the centrum semiovale $(37.75 \%$ units $v$ $37.57 \%$ units, $P<0.05)$ and parietooccipital white matter $(37 \cdot 67 \%$ units $v$ $37.43 \%$ units, $P<0.05)$. No relation between such interhemispheric MTR differences and handedness was noted.

Conclusions-Magnetisation transfer imaging shows significant age related changes in normal brain white matter. In addition to regional variations in MTR in the normal brain, there seem to be small but significant variations in MTR between the cerebral hemispheres. It is important to consider such normal variations when evaluating MTR in pathological states.

(F Neurol Neurosurg Psychiatry 1997;62:223-228)

Keywords: magnetisation transfer ratio; normal brain white matter; aging
Magnetic resonance imaging (MRI) provides a sensitive, non-invasive, and safe tool for elucidating pathological change, and as such has been used to evaluate a considerable number of disorders of the CNS. Contrast in conventional MRI sequences mainly results from differences in relaxation properties and proton density of mobile (free) water protons. Conventional MRI thus has a high sensitivity in detecting pathological tissue but pathological specificity is poor: a similar signal change (due to altered water content) may result from disease processes such as loss of normal tissue (demyelination and neuronal damage), oedema, inflammation, infection, infiltration by tumour cells, and gliosis. There also exists a separate pool of water protons tightly bound to macromolecular structures such as proteins and lipid membranes, which, by virtue of their very short $\mathrm{T} 2$ relaxation times are essentially invisible to conventional MRI sequences. Magnetisation transfer (MT) imaging is a relatively new technique which probes the bound proton pool and thus may have potential for in vivo differentiation between such pathological entities.

By exploiting differences in the resonant properties of free and bound protons, a saturation prepulse can be utilised to selectively saturate the bound proton pool. The saturation is transferred by chemical exchange and other processes such as spin diffusion resulting in a decrease in the measurable free proton signal with consequent reduction in tissue signal intensity. The magnitude of this effect, the magnetisation transfer ratio (MTR), therefore reflects the amount and nature of macromolecular structure present in a given volume. ${ }^{1}$ There is currently intense research effort being directed towards the development of models to provide a detailed quantitative understanding of magnetisation transfer effects.

Measurement of MTR is increasingly being used in the evaluation of disorders of the CNS. ${ }^{2-10}$ There is also a potential role in multiple sclerosis for assessing the efficacy of new experimental therapies. ${ }^{11}$ It is therefore important in interpreting such studies to understand how MTR may vary with normal aging and whether a significant effect of sex exists. In addition, for those studies investigating unilateral pathology (using the normal side as a control), it is important to know whether there is any relevant interhemispheric variation in the normal brain. The aim of this study was to evaluate such variations in normal brain white matter. 
Calculated MTR images showing regions of interest for mean MTR measurement.
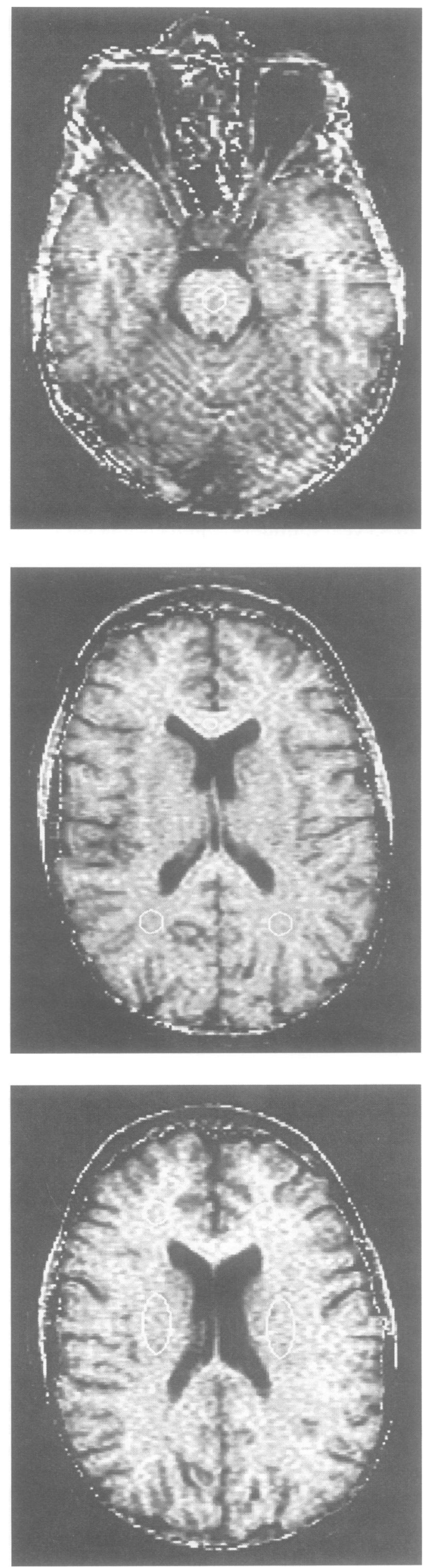

\section{Subjects and methods}

\section{SUBJECTS}

Forty one healthy volunteers (aged between 16 and 55 years) were recruited as follows with the sexes matched for age: 16-25 years (five men, five women), 26-35 years (five men, six women), 36-45 years (five men, five women), and 46-55 years (five men, five women). The mean age of the male group $(n=20)$ studied was 36.7 years and that of the female group ( $\mathrm{n}$ $=21$ ) was 34.4 years. All subjects were entered into the study on the basis of a normal neurological history.

Handedness was assessed in all subjects using a questionnaire devised and validated by Annett. ${ }^{12}$ This assesses hand preference for 12 manual tasks. For the purposes of this study, we assigned a value of +2 points for each task performed preferentially with the right hand and -2 points for the left hand. Handedness was assigned according to an arbitrary total score (left handed between -24 and -9 , ambidextrous between -9 and +9 , right handed between +9 and +24 ).

All subjects gave written, informed consent to participate in the study, which had been approved by the ethics committee of the Institute of Neurology and the National Hospital for Neurology and Neurosurgery.

MRI PROTOCOL

All subjects were imaged using a 1.5 Tesla MRI scanner with standard quadrature headcoil (Signa, GE Medical Systems, Milwaukee, Wisconsin). A dual spin echo sequence (TR 1730, TE31/80, 28 contiguous $5 \mathrm{~mm}$ axial slices, $3 / 4 \mathrm{Nex}, 256 \times 128$ matrix, $240 \times 240$ $\mathrm{mm}$ field of view) was performed both with and without presaturation pulses to saturate the broad resonance of immobile macromolecular protons. This was adapted from a previous sequence developed by Barker et al..$^{13}$ The applied presaturation pulse was a Hamming apodised three lobe sinc pulse, with a duration of $16 \mathrm{~ms}$ and a peak amplitude of $23.2 \mu \mathrm{T}$ (giving a nominal bandwidth of $250 \mathrm{~Hz}$ ), applied $1 \mathrm{kHz}$ off water resonance. The energy deposited by this pulse provided measurable differences between saturated and unsaturated images and ensured a good signal to noise ratio in the calculated MT image. To ensure exact coregistration of the pixels on saturated and non-saturated images, scans with and without presaturation were interleaved for each TR period. ${ }^{13}$ The total time of acquisition was $17 \cdot 7$ minutes.

In all subjects, MTR was calculated on a pixel by pixel basis from the formula:

$$
\left[M_{0}-M_{s}\right] /\left[M_{o}\right] \times 100 \% \text { units (pu) }
$$

where $M_{\mathrm{s}}$ and $M_{\mathrm{o}}$ represent signal intensities with and without the saturation pulse respectively. Calculated MT images were displayed on a Sun workstation using image display software. ${ }^{14}$ All MTR analysis was carried out by one of us (NCS) blinded to subject identity.

Regions of interest were outlined on the non-MT, T2 weighted (TE 80) images. Care was taken to include only white matter, leaving 
Table 1 Regional variation of MTR measurement in normal brain white matter (mean (SEM) \% units)

\begin{tabular}{ll}
\hline & $M T R$ \\
\hline Corpus callosum (genu) & $40 \cdot 56(0 \cdot 13)$ \\
Pons & $38 \cdot 93(0 \cdot 19)$ \\
Left frontal & $39 \cdot 50(0 \cdot 11)$ \\
Right frontal & $39 \cdot 35(0 \cdot 12)$ \\
Left centrum semiovale & $37 \cdot 75(0 \cdot 09)$ \\
Right centrum semiovale & $37 \cdot 57(0 \cdot 11)$ \\
Left parieto-occipital & $37 \cdot 79(0 \cdot 15)$ \\
Right parieto-occipital & $37 \cdot 55(0 \cdot 12)$ \\
\hline
\end{tabular}

a surrounding rim of white matter (to minimise partial volume effect from grey matter or CSF). The adjacent slices were checked to ensure that all regions of interest were fully surrounded by white matter. No regions of interest included minor areas of T2 hyperintensity, a "normal" finding in healthy adults. For all subjects, regions of interest were identical in size and shape for each anatomical area studied. Mean MTR measurements were then taken from the corresponding regions on calculated MTR images (figure). In both cerebral hemispheres regions were chosen in the centrum semiovale $\left(216 \mathrm{~mm}^{2}\right)$, frontal white matter $\left(70 \mathrm{~mm}^{2}\right)$, and parieto-occipital white matter $\left(70 \mathrm{~mm}^{2}\right)$. In addition, midline regions were also evaluated in the genu of the corpus callosum $\left(23 \mathrm{~mm}^{2}\right)$ and pons $\left(70 \mathrm{~mm}^{2}\right)$.

\section{STATISTICAL ANALYSIS}

Statistical analysis was carried out using Levene's test for equality of variances, Student's $t$ test, paired $t$ test, and Spearman rank correlation coefficient as appropriate.

\section{Results}

Significant regional variations in MTR values were noted throughout the white matter in the

Table 2 Effect of sex on regional MTR measurements (mean (SEM) \% units)

\begin{tabular}{lll}
\hline & Male $(n=20)$ & Female $(n=21)$ \\
\hline Corpus callosum (genu) & $40 \cdot 41(0 \cdot 20)$ & $40 \cdot 70(0 \cdot 17)$ \\
Pons & $39 \cdot 15(0 \cdot 29)$ & $38 \cdot 72(0 \cdot 24)$ \\
Left frontal & $39 \cdot 45(0 \cdot 18)$ & $39 \cdot 55(0 \cdot 14)$ \\
Right frontal & $39 \cdot 43(0 \cdot 15)$ & $39 \cdot 28(0 \cdot 20)$ \\
Left centrum semiovale & $37 \cdot 87(0 \cdot 12)$ & $37 \cdot 63(0 \cdot 12)$ \\
Right centrum semiovale & $37 \cdot 65(0 \cdot 16)$ & $37 \cdot 50(0 \cdot 17)$ \\
Left parieto-occipital & $37 \cdot 56(0 \cdot 26)$ & $38 \cdot 01(0 \cdot 14)$ \\
Right parieto-occipital & $37 \cdot 45(0 \cdot 17)$ & $37 \cdot 64(0 \cdot 17)$ \\
\hline
\end{tabular}

Table 3 Interhemispheric variation of white matter MTR values (mean (SEM) \% units) in all 41 subjects

\begin{tabular}{llll}
\hline & $\begin{array}{l}\text { Left } \\
\text { hemisphere }\end{array}$ & $\begin{array}{l}\text { Right } \\
\text { hemisphere }\end{array}$ & $\begin{array}{l}\text { Pvalue } \\
\text { (paired t test) }\end{array}$ \\
\hline Frontal & $39.50(0.11)$ & $39.35(0.12)$ & - \\
Centrum semiovale & $37.75(0.09)$ & $37.57(0.11)$ & $<0.05$ \\
Parieto-occipital & $37.79(0.15)$ & $37.55(0.12)$ & $<0.05$ \\
\hline
\end{tabular}

Table 4 Effect of age on white matter MTR measurements (mean (SEM) \% units)

\begin{tabular}{|c|c|c|c|}
\hline & $16-35 y$ & $36-55 y$ & $\begin{array}{l}\text { P value } \\
\text { (student's } t \text { test) }\end{array}$ \\
\hline $\begin{array}{l}\text { Frontal }{ }^{\star} \\
\text { Centrum semiovale*} \\
\text { Parieto-occipital }^{\star} \\
\text { Corpus callosum (genu) } \\
\text { Pons }\end{array}$ & $\begin{array}{l}39.65(0.10) \\
37.83(0.09) \\
37.88(0.13) \\
40.82(0.16) \\
38.97(0.24)\end{array}$ & $\begin{array}{l}39 \cdot 18(0.11) \\
37.49(0 \cdot 10) \\
37 \cdot 44(0 \cdot 14) \\
40 \cdot 28(0 \cdot 20) \\
38 \cdot 89(0.30)\end{array}$ & $\begin{array}{l}<0.005 \\
<0.02 \\
<0.05 \\
<0.05\end{array}$ \\
\hline
\end{tabular}

^For the hemispheric regions, both right and left hemisphere values for MTR are included in the analysis. normal brain (table 1 ). In the 41 subjects studied, highest values were found in the corpus callosum $(40 \cdot 1 \mathrm{pu})$. This was significantly higher than any other region studied $(P<$ 0.001 , paired $t$ test). For the three hemisphere regions studied, significantly higher MTR values were seen in frontal white matter $(P<$ 0.001 , paired $t$ test).

MTR values for each region were evaluated between the male and female subjects (Levene's test for equality of variances and Student's $t$ test) and no significant male/ female difference was noted for any region (table 2).

White matter regions (frontal, centrum semiovale, and parieto-occipital) in the right and left sides of the brain were compared in all normal volunteers (table 3). In all these regions, MTR values were higher in the left hemisphere. In frontal white matter, this difference was not significant (paired $t$ test). Differences between the hemispheres were significant in the centrum semiovale $(P<0.05$, paired $t$ test) and parieto-occipital white matter $(\mathrm{P}<0.05$, paired $t$ test). Assessment of handedness using the Annett handedness questionnaire $^{12}$ showed 35 normal volunteers to be right handed, one ambidextrous, and five left handed. For the 35 right handed subjects, higher MTR values were consistently seen in the left hemispheric regions (frontal white matter: 39.47 v 39.30; centrum semiovale: $37.69 v 37.51$; parieto-occipital white matter: $37.67 v 37.43)$. Such differences were significant in the centrum semiovale $(P=0.05$, paired $t$ test). Although left hemispheric MTR values were also consistently higher than on the right side in the five left handed subjects (frontal white matter: $39.64 v 39.54$; centrum semiovale: $38.24 v$ 37.92; parieto-occipital white matter: $38.46 v 38.36$ ), none of these differences was significant on paired $t$ test analysis.

Initially, to assess the effects of age on white matter MTR measurements, the normal volunteers were divided into four groups (16-25, $26-35,36-45,46-55$ years). All individual regions were evaluated for these groups and no significant association with age was immediately apparent (Student's $t$ test). Because the number of subjects in each group was small, these results were further evaluated dividing the population into two larger age groups (16-35 years, $\mathrm{n}=21$ and $36-55$ years, $\mathrm{n}=$ 20). Five white matter regions (frontal, centrum semiovale, parieto-occipital, pons, and the genu of the corpus callosum) were evaluated. For the hemispheric regions, both right and left hemispheric MTR values for each subject were considered in the analysis. The two age groups were compared for each region by Student's $t$ test. Significantly lower MTR values were noted in all regions except for the pons in the older age group compared with the 16 to 35 year group (table 4 ). The relation between age and MTR was further evaluated by Spearman's rank correlation coefficient analysis. A significant inverse correlation was noted between age and MTR for all regions studied with the exception of the pons (frontal 
white matter: $r=-0.34, \mathrm{P}<0.005$; centrum semiovale: $r=-0.35, \mathrm{P}<0.005$; parietooccipital white matter: $r=-0.25, \mathrm{P}<0.05$, genu of the corpus callosum: $r=-0.32, \mathrm{P}<$ $0.05)$. For this analysis, both right and left hemispheric MTR measurements were included.

\section{Discussion}

Tissue contrast mechanisms in conventional MRI rely on three major characteristics of free water protons - namely, their density, spin-lattice (T1), and spin-spin (T2) relaxation properties. As such, conventional MRI techniques lack specificity in distinguishing different pathological processes affecting the CNS. MT imaging provides a different mechanism of contrast which exploits the properties of those protons bound to macromolecules that are not visualised with conventional techniques. ${ }^{1}$ Measurement of MTR may be more specific in detecting structural damage in white matter of the brain, especially myelin loss. ${ }^{23} 715-17$ This is particularly relevant to the assessment of pathological processes occurring in demyelinating disorders such as multiple sclerosis, in which the amount of magnetisation transfer is affected by frank destruction of the myelin sheaths. ${ }^{2}$ To assess such a new technique in pathological states, it is important to understand how values for MTR may vary in the white matter of normal brain. In this study we have looked at the normal regional variations for MTR in brain white matter and assessed the effects of sex and early normal aging. The possible mechanisms of such variations and their implications are discussed.

To obtain MTR measurements in this study we chose a spin echo based sequence with interleaved MT pulses. This was based on a sequence originally developed by Barker et al. ${ }^{13}$ By decreasing the effect of motion (which may affect image subtraction and therefore accurate calculation of MTR) this technique has theoretical advantages over the more conventional gradient echo based sequences used in many centres. Because the MT pulse is interleaved this is also helpful in designating the region of interest using the $\mathrm{T} 2$ weighted image. This has, in our opinion, two advantages. Firstly, the T2 weighted image provides good differentiation between grey and white matter and allows accurate placement of the region of interest in white matter, reducing partial volume effects from grey matter or CSF. Secondly, because contrast mechanisms differ between T2 weighted and calculated MTR images, the assessor is blinded to the appearance of the calculated MTR image when placing the region of interest (while accurate coregistration is ensured).

In this study, the highest value for MTR was seen in the genu of the corpus callosum, as compared with white matter regions in the cerebral hemispheres and pons $(P<0.001$, Student's $t$ test; table 1). In addition, frontal white matter showed significantly higher MTR values than those found in the centrum semiovale and parieto-occipital white matter $(\mathbf{P}<$
$0 \cdot 001$, Student's $t$ test). These regional variations are in agreement with previous studies evaluating MTR in the normal brain. ${ }^{31318}$ Possible explanations for such MTR variation in the different regions include possible differences in fibre packing density, degree of myelination, tissue hydration, and vascularity. The finding of high MTR values in the corpus callosum would seem to correlate with the histological appearance of a large number of myelinated fibres (about 300 million) in this structure. ${ }^{19}$ Our results have also shown no effect of sex on measurements of MTR for any region studied (table 2). This is in agreement with a previous study. ${ }^{18}$

In addition to regional intrahemispheric MTR variations, we have found significant interhemispheric differences (table 3). Higher MTR values were seen in the left hemisphere for all three regions studied (centrum semiovale, parieto-occipital, and frontal white matter), although this difference was not significant in frontal regions. In a previous report, Mehta et al ${ }^{18}$ did not show white matter asymmetry. Several factors may account for this discrepancy. Firstly, our study has relied on an interleaved spin echo sequence with predominant proton density weighting as opposed to the separate acquisition of $M T$ and non-MT prepared T1 weighted (TR $800 \mathrm{~ms}$ ) data performed by Mehta et al. ${ }^{18}$ The interleaved sequence has potential advantages of image coregistration between MT and non-MT images, theoretically allowing more accurate subtraction and consequent MTR calculation. ${ }^{13}$ Secondly, it is less likely that $\mathrm{T} 1$ relaxation properties of tissue will affect MTR values obtained when a longer TR of $1730 \mathrm{~ms}$ is used. Thirdly, the advantages of a coregistered T2 weighted image for placing regions of interest have already been discussed. Fourthly, our study has relied on using controls with no neurological symptoms or history of neurological disease, as opposed to retrospective assessment of patients in whom imaging was normal and a neurological disease was considered unlikely..$^{18}$ All these factors may account for the wider ranges of MTR found by Mehta et $a l$, and which in turn may have obscured small interhemispheric differences. Using a similar technique to that of our study, Barker et al ${ }^{13}$ showed occipital asymmetry in 10 normal subjects, although with higher MTR values in the right hemisphere.

Although artefactual causes for such interhemispheric MTR differences may exist and are difficult to exclude (for example, coil asymmetry with consequent inhomogeneities in the MT presaturation pulse), a natural asymmetry in the brain is well recognised. It is widely accepted that the two hemispheres of the brain are functionally specialised for different cognitive tasks. On a cellular and morphological level, various asymmetries exist in human cortical structures such as the planum temporale ${ }^{2021}$ and parietal structures. ${ }^{22}$ Further evidence from in vivo imaging studies includes planum temporale and parietal operculum asymmetry noted with $\mathrm{MRI}^{23}$ and hemispheric width, lateral ventricle, sylvian fissure, and 
occipital petalia asymmetry all noted with CT. ${ }^{24}{ }^{25}$ Possible explanations for interhemispheric MTR asymmetry could be differences in fibre packing density, degree of myelination, or phospholipid metabolism between the two hemispheres. Interestingly, in rats, total phospholipid content and sphingomyelin concentrations have been shown to be higher in the left hemisphere with significant asymmetries in cortical phospholipid metabolism. ${ }^{26}$

Many necropsy and in vivo studies have noted a relation between handedness and hemispheric asymmetry, with reduced asymmetry in left handers. ${ }^{27}$ In the present study we found that, for right handed subjects, MTR values in parieto-occipital white matter were significantly higher in the left hemisphere, whereas no significant asymmetry was found in left handed subjects; no firm conclusions can be drawn, however, because of the very small number of left handed subjects studied.

Our results have shown that there is a relation between MTR and age, increasing age being associated with reduced MTR values in the corpus callosum and all three hemispheric white matter regions studied. We are not aware of other reports of MTR reductions with age. Mehta et al found no discernible MTR variations between 21 and 80 years of age, ${ }^{18}$ but as already stated, there are several reasons why the small differences we found might not have been detected using their protocol. This cross sectional finding should be interpreted with caution; it is possible that unknown environmental factors may play a part in the differences between younger and older subjects and that it is not solely an effect of aging itself. To be more certain, a longitudinal study is required to show intraindividual changes with time. However, cross sectional MRI studies have shown significant relations between brain atrophy and age in similar age groups to those studied here, ${ }^{28} 29$ and one longitudinal MRI study in 11 controls (mean age 55 years) has shown a small but significant reduction in brain volume over a single year. ${ }^{30}$ It is probable that such age related variation in MTR is real and we postulate that this may reflect either structural changes in brain white matter (for example, neuronal loss, consequent myelin loss, changes in glial tissue, or altered water content) or changes in phospholipid metabolism with increasing age.

The absolute MTR measurements found depend on the exact imaging sequence variables used ${ }^{31}{ }^{32}$ which accounts for differences in MTR measurement in normal white matter noted between our study and previous studies. $^{231318}$ Differences in performance between scanners might also influence MTR measurements even when the sequence is identical. The "normal values" we report are therefore only applicable to one particular scanner using one particular technique. Further work is needed to develop MT imaging protocols that lead to comparable MTR values being obtained on different scanners. ${ }^{32}$ Such progress will be of value for multicentre treatment trials or natural history studies.

In conclusion, MTR measurement seems a robust in vivo technique with potential applications for evaluating structural changes in brain white matter occurring both in health and disease states and may play an important part in assessing response to therapies in demyelinating illnesses. We have shown regional and age related variations in MTR of white matter in the normal brain. It is important to consider such findings when interpreting MTR changes in pathological states.

We thank Professor WI McDonald for helpful discussions. The NMR Research Unit is supported by a generous grant from the Multiple Sclerosis Society of Great Britain and Northern Ireland.

1 Wolff SD, Balaban RS. Magnetisation transfer contrast (MTC) and tissue water proton relaxation in vivo. Magn Reson Med 1989;10:135-44.

2 Dousset V, Grossman RI, Ramer KN, Schnall MD, Young LH, Gonzalez-Scarano F, et al. Experimental allergic encephalomyelitis and multiple sclerosis: lesion characterisation with magnetization transfer imaging. Radiology terisation with ma

3 Gass A, Barker GJ, Kidd D, Thorpe JW, MacManus D Brennan A, et al. Correlation of magnetization transfer ratio with clinical disability in multiple sclerosis. Ann Neurol 1994;36:62-7.

4 Tomiak MM, Rosenblum JD, Prager JM, Metz CE. Magnetization transfer: a potential method to determin the age of multiple sclerosis lesions. AFNR Am Neuroradiol 1994;15:1569-74.

5 Hiehle JF, Grossman RI, Ramer KN, Gonzales-Scarano F, Cohen JA. Magnetization transfer effects in MR-detected multiple sclerosis lesions: comparison with gadoliniumenhanced spin-echo images and non-enhanced T1weighted images. AfNR Am fournal Neuroradiol 1995;16: 69-77.

6 Ordidge RJ, Helpern JA, Knight RA, Quing Z, Welch KMA. Investigation of cerebral ischaemia using magnetization transfer (MTC) MR imaging. Magn Reson Imaging 1991;9:895-902.

7 Dousset V, Armand JP, Degrèze $P$, Mièze $S$, Lafon $P$ Caillé JM. Progressive multifocal leucoencephalopathy studied by magnetization transfer imaging [abstract]. Proceeding of the Society of Magnetic Resonance and the European Society for Magnetic Resonance in Medicine and Biology, Nice, France, 1995;284.

8 Boorstein JM, Wong KT, Grossman RI, Bolinger L, McGowan JC. Metastatic lesions of the brain: imaging with magnetization transfer. Radiology 1994;191: 799-803.

9 Wong KT, Grossman RI, Boorstein JM, Lexa FJ, McGowan JC. Magnetization transfer imaging of periventricular hyperintense white matter in the elderly. periventricular hyperintense white matter
AfNR Am $₹$ Neuroradiol 1995;16:253-8.

10 Tofts PS, Sisodiya S, Barker GJ, Webb S, MacManus D, Fish D, Shorvon S. MR magnetization transfer measurements in temporal lobe epilepsy: a preliminary study AFNR Am F Neuroradiol 1995;16:1862-3.

11 Miller DH, Albert PS, Barkhoff F, Francis G, Frank JA Hodgkinson $S$, et al. Guidelines for the use of magnetic resonance techniques in monitoring the treatment of multiple sclerosis. Ann Neurol 1996;39:6-16.

12 Annett $M$. Lefi, right, hand and brain: the right shift theory. London: Lawrence Erlbaum Associates, 1985:183-204.

13 Barker GJ, Tofts PS, Gass A. An interleaved sequence for accurate and reproducible clinical measurement of magnetization transfer ratio. Magn Reson Imaging 1996;14: 403-11.

14 Plummer DL. DispImage: a display and analysis tool for medical images. Revista Di Neuroloradiologica 1992;5: 489-95.

15 Thorpe JW, Barker GJ, Jones SI, Moseley I, Losseff N, MacManus DG, et al. Magnetisation transfer ratios and transverse magnetisation decay curves in optic neuritis: correlation with clinical findings and electrophysiology. $f$ Neurol Neurosurg Psychiatry 1995:59:487-92.

6 Dousset V, Brochet B, Vital A, Gross C, Benazzouz A Boullerne A, et al. Lysolecithin-induced demyelination in primates: preliminary study with MR and magnetization transfer. AfNR Am ₹ Neuroradiol 1995;16:225-31.

17 Silver NC, Barker GJ, Macmanus DG, Thorpe JW, Howard R, DH Miller. Decreased magnetisation transfer ratio due to demyelination: a case of central pontine myelinolysis. $f$ Neurol Neurosurg Psychiatry 1996;61: myelin 9 .

18 Mehta RC, Pike B, Enzmann DR. Magnetization transfer MR of the normal adult brain. AfNR Am $\mathcal{F}$ Neuroradiol 1995;16:2085-91.

19 Barr ML, Kiernan JA. The human nervous system: an anatomical viewpoint. Philadelphia: JB Lippincott, 1993 $251-68$.

20 Geschwind N, Levitsky W. Human brain: left-right asymmetries in temporal speech region. Science 1968;161: 186-7.

21 Wada JA, Clarke R, Hamm A. Cerebral hemispheric asymmetry in humans. Arch Neurol 1975;32:239-46.

22 Eidelberg D, Galaburda AM. Inferior parietal lobule: divergent architectonic asymmetries in the human brain. Arch
Neurol $1984 ; 41: 843-52$. 
23 Habib M, Robichon F, Levrier O, Khalil R, Salamon G. Diverging asymmetries of temporo-parietal cortical areas: Diverging asymmetries of temporo-parietal cortical areas: a reappraisal of Gesch

24 Le May M, Kido DK. Asymmetries of the cerebral hemispheres on computerised tomograms. F Comput Assist Tomog 1978;2:471-6.

25 Shapiro R, Galloway SJ, Shapiro MD. Minimal asymmetry of the brain: a normal variant. AfR Am $\mathcal{F}$ Roentgenol 1986;47:753-6.

26 Pediconi MF, Barrantes FJ. Brain asymmetry in phospholipid polar head group metabolism: parallel in vivo and in vitro studies. Neurochem Res 1990;15:25-32.

27 Beaton A. Left sidelright side: a review of laterality research. London: Batsford Academic and Educational, 1985 : 143-54.

28 Blatter DD, Bigler ED, Gale SD, Johnson SC, Anderson $\mathrm{CV}$, Burnett BM, et al. Quantitative volumetric analysis of brain MR: normative database spanning five decades of life. AFNR Am $\mathcal{F}$ Neuroradiol 1995;16:241-51.

29 Condon B, Grant R, Hadley D, Lawrence A. Brain and intracranial cavity volumes: in vivo determination by MRI. Acta Neurol Scand 1988;78:387-93.

30 Fox NC, Freeborough PA, Rossor MN. Visualisation and quantification of rates of atrophy in Alzheimer's disease. Lancet 1996;348:94-7.

31 Hajnal JV, Baudouin CJ, Oatridge A, Young IR, Bydder GM. Design and implementation of magnetization transfer pulse sequences for clinical use. $\mathcal{F}$ Comput Assist Tomogr 1992;16:7-18.

32 Berry I, Barker G, Barkhoff F, Campi A, Dousset V, Franconi JM, et al. A multicentre measurement of magnetisation transfer ratio in normal white matter. Procedings of the sation transfer ratio in normal white matter. Proceedings of the New York: 1996:536.

\section{HISTORICAL NOTES}

\section{Marshall Hall and the concepts of reflex action}

In Tractatus Homine (1662) Descartes introduced the concept of mind-body dualism, and contrary to all current thought, asserted that automatic actions occurred independently of the soul. In the early 19th century the cord, like the brain, was thought to have a psychic function of soul. Reflex action was considered a manifestation of an informing spirit resident in the organism (Sherrington ${ }^{1}$ ).

The physiological groundwork for neural function evolved slowly. Jan Swammerdam (1637-80) had discovered that isolated nerves were irritable if still attached to a muscle. Bell ${ }^{2}$ and Magendie distinguished sensory from motor nerves. Albrecht von Haller showed that muscle itself also was irritable. And, Robert Whytt's experiments showed that the spinal cord was needed for the "sympathy" between different parts of the body; and he clearly described the stretch reflex in 1763. Procháska had realised that motor and sensory nerves were connected, but related this to some latent, intrinsic force: "the vis nervosa is latent, nor excites action of the nervous system until excited by an applied stimulus ..."

But it was the controversial work of Marshall Hall in 1833 that plainly showed the reflex function-an "excito-motory system" nerves in animals after removal of the brain. This ended the misconceptions that the soul and psychic functions resided in the cord as well as in the brain. Since Hall failed to obtain a hospital appointment, his studies were based on experiments in his own home on frogs, lizards, eels, snakes, and turtles. His discoveries began whilst examining the capillary circulation:

"I incidentally observed a remarkable phenomenon; the separated tail of the eft [newt] moved on being irritated by the point of the scalpel... I conceived it impossible that any such phenomenon should exist in nature without such connection, and I resolved to pursue the subject."

The demonstration by Golgi and Cajal of a network of neurons ("retia nervosa diffusa") that mediated complex movements, formed a foundation for observations of spasticity, clonus, and tendon reflexes by Charcot and Vulpian, Erb and Westphal, which were delayed until 1860-1880.

Marshall Hall recognised and described ${ }^{5}$ that the cerebrum was the source of voluntary motion, the medulla oblongata was the source of respiratory motion, and the spinal cord was the middle arc of reflex function. He then described reflex activity:

"There is a fourth which subsists, in part, after voluntary and respiratory motions have ceased by removal of the cerebrum and medulla oblongata, and which is attached to the medulla spinalis, ceasing itself when this is removed and leaving the irritability undiminished. In this kind of muscular motion, the motive influence does not originate in any part of the central nervous system, but at a distance from that centre: it is neither spontaneous in its action, nor direct in its course; it is, on the contrary, excited by the application of appropriate stimuli, which are not, however, applied immediately to the muscular or nervo-muscular fibre, but to certain membranous parts, whence the impression is carried to the medulla, reflected, and reconducted to the part impressed, or conducted to a part remote from it, in which muscular contraction is effected."

His 1837 memoir $^{3}$ was critical of the deductions of Procháska and many other physiologists, but he allowed that Sir Gilbert Blane (1749-1834), physician to St Thomas's hospital came nearest the truth in his statement: . . "instinctive or automatic motions, can be exerted without the intervention of the sensorium commune, without sensation or consciousness". Blane had observed an acephalic monster, and noted that a decapitated bee could still sting.

In his Coonian lecture ${ }^{6}$ he introduced the term diastaltic, referring to the reflex action through the cord. Diastaltic was "congeric with peristaltic". $\mathrm{He}$ added:

"I observed that [for a spinal reflex] the following anatomical relations are essential:

1. A nerve leading from the point or part irritated, to and into the spinal marrow;

2. The spinal marrow itself; and

3. A nerve, or nerves, passiong out or from the spinal marrow, - all in essential relation or connection with each other."

Despite many objections to his argumentative and captious presentations and to the novelty of his conclusions, his work was eventually accepted by the neurological hierarchy. It is not without interest that he was made FRS in 1832, but Fellowship of the Royal College of Physicians was not granted until 1841. JMS PEARCE
304 Beverley Road, Anlaby, Hull,

1 Sherrington C. The integrative action of the nervous system. Cambridge: Cambridge University Press, 1947: 319.

Pearce JMS. Sir Charles Bell (1774-1842). $\mathcal{F} R$ Soc Med 1993;86:353-4.

3 Hall M. Memoirs on the nervous system. Memoir II. On the true spinal marrow and the excito-motory system. London: true spinal marrow and the excito-motory system. London:
Sherwood, Gilbert, and Piper, 1837. [Read to the Royal Sherwood, Gilbert, and Piper, 1837.

4 Hall $M$. On the diseases and derangements of the nervous system. London: 1841 .

5 Hall $M$. On the reflex function of the medulla oblongata and the medulla spinalis. Philos Trans $R$ Soc Lond $B$ 1833;123:635-65.

6 Hall M. Synopsis of the diastaltic nervous system: or the system of the spinal marrow and its reflex arcs, as the nervous agent in all the functions of ingestion and of egestion in the animal oeconomy. London: J Mallett, 1850. 